\title{
POROUS SILICON - NEW LUMINESCENT MATERIAL OR LUMINESCENT DEFECT?
}

\author{
M. Mizuta \\ Fundamental Research Laboratories, NEC Corporation, Tsukuba, Ibaraki 305, Japan
}

The recent topic of visible luminescent porous $\mathrm{Si}$ is reviewed with an emphasis on luminescent mechanism. Among various mechanisms proposed, size quantization plays some important role and is experimentally observable but it would not be a key entity which emits visible light in dispute. The role of oxygen is found to be very important and this is a key constituent for the luminescent substance. A possible mechanism of localized electron-hole pair recombination nature for porous $\mathrm{Si}$ involving oxygen is discussed.

PACS numbers: 78.66.-w

\section{Introduction}

Porous silicon has been known over 30 years and it was first reported during electropolishing of $\mathrm{Si}$ in aqueous hydrofluoric acid. Porous nature is quite attractive for the present and future Si microprocess technology, considering that it is a low-temperature processing. Porous $\mathrm{Si}$ and its fabrication technology have been accounted for the possible use in electronic isolation, such as SOI (silicon on insulator), capacitive component using microtrench, three-dimensional interconnection by buried conductive layer made of silicide which is also useful for stress relaxed metallization, etc. Furthermore, porous $\mathrm{Si}$ can be useful for other industrial purposes, such as chemical sensing, catalytic materials, etc. Its luminescent nature, however, has attracted an extensive interest quite recently, triggered by the report of Canham [1]. The reasons for this vast interest are, from one side, due to possible monolithic integration of Si photonic and electronic circuits and, from the other side, due to exploration of the mechanism of new efficient, visible luminescence from silicon, which has indirect band gap in the infrared when it is bulk material. Size quantization resulting from small Si clusters (dots) or $\mathrm{Si}$ one-dimensional wires formed during anodization of $\mathrm{Si}[1,2]$ has been first proposed as the mechanism for the visible light emission from this material. However, upon accumulating experimental results, other models accounting for the mechanism of visible luminescence have been proposed, including polysilane structure, siloxene and its derivative structure at the (inner) surface of the pore, and localized surface states, such as $\mathrm{Si}-\mathrm{H}$ or $\mathrm{Si}-\mathrm{O}$. Resemblance of photoluminescence (PL) to that from 
amorphous Si has also been pointed out. Up to now, however, no definite model has been accepted. Several recent annealing studies and other characterizations shed some light on the mechanism of porous $\mathrm{Si}$ luminescence where $\mathrm{Si}-\mathrm{O}$ bond or its containing compound is quite important although the size quantization effect could not be discarded.

This article discusses and summarizes reported experimental and theoretical results of porous $\mathrm{Si}$ with a particular focus on its luminescence mechanism and proposes a plausible picture for the visible luminescence from porous $\mathrm{Si}$.

\section{General features of porous $\mathrm{Si}$ : experimental observations}

\subsection{Phololuminescence and related characterization}

The room temperature photoluminescence in the visible region from porous Si has been first reported by Canham. Porous Si produces light quite efficiently and it is very easy to fabricate. These are the reasons for its attracting wide-spread interest besides application interests. An absolute value of quantum efficiency for the light emission is sometimes difficult to measure but it is reported in the order of several percent in photoluminescence [3], which should be compared to the same several percent efficiency for presently existing $\mathrm{GaP}$ light emitting diode, where the efficiency is measured by the current injection (photoluminescence quantum efficiency for red light emitting GaP:Zn,O has been reported to be $\approx 20 \%$, though). Indirect band gap materials such as $\mathrm{GaP}$ and $\mathrm{Si}$, have typically very low luminescence efficiency, because the momentum conservation upon electron-hole recombination is difficult to be fulfilled. In $\mathrm{GaP}$, efficient luminescence is a result of the introduction of isoelectronic trap, which enhances the recombination rate by three orders of magnitude as compared with a typical shallow impurity, for which the Fourier component of spatially localized wave function of the isoelectronic trap extends to $k=0$, where the valence band maximum exists [4]. Therefore, a similar effect should take place in the case of porous $\mathrm{Si}$, if $\mathrm{Si}$ itself is a luminescent medium.

The fabrication of the pore structure in Si is simply an electrochemical anodization of Si in aqueous IF (typically together with ethyl alcohol) [5]. Pore formation and its structure are critically dependent on anodization current, substrate type and external excitation during anodization, such as light illumination [6]. Thickness of the pore-structured part is clearly dependent on time of anodization and it can extend to several hundred micrometers. Fabrication of laterally as well as vertically uniform layer requires some tricks but luminescent porous Si can easily be obtained.

The typical photoluminescence peaks between $1.6-1.9 \mathrm{eV}$, depending on fabrication conditions, with its half width of $0.3-0.4 \mathrm{eV}$ regardless of its peak position [6-8]. For the $n$-type substrate, pore formation requires light illumination during anodization and its photoluminescence is typically located in higher energy as compared to $p$-type substrate [6]. Absorption of the porous $\mathrm{Si}$ is somewhat difficult to measure because of the existence of Si substrate. During anodization, by changing the current density to switch from pore formation mode to electropolishing mode, 
a free-standing porous Si layer can be obtained. Using such a film, absorption can be measured and it is found to be dependent on the size of Si microcluster whose size is typically evaluated by TEM (transmission electron microscope) [9] and Raman scattering [10]; a blue shift of the absorption edge occurs for films containing smaller size crystal [11]. The luminescence excitation, which generally coincides with absorption in semiconductor, however, begins at $2.4 \mathrm{eV}$ and its threshold stays relatively constant, that is, it does not depend on size of microcrystals [8]. A typical example of PL peaked at $1.7 \mathrm{eV}$ and absorption begins at $1.5 \mathrm{eV}$ and luminescence excitation begins at $2.4 \mathrm{eV}$. This apparent contradiction between excitation and absorption is probably due to the non-homogeneous nature of porous Si material, in other words, porous Si of the gross is not necessarily the same substance which gives luminescence in the visible. If the reported excitation spectrum really represents the excited state of the luminescent center, the large difference of PL and PL excitation peaks apparently shows the existence of the large Stokes shift which indicates the strong electron-phonon coupling. The large half width of PL could be accounted for by the large electron-lattice interaction, but the temperature dependence of PL half width does not appreciably change with lowering temperature. In a typical large electron-phonon coupling system, the half width of PL is expected to follow the relation, $W^{2}(T)=W^{2}(0) \operatorname{coth}(h \omega / 2 k T)$, but no evident report has been made for this relationship and rather, the half width is insensitive to temperature. This suggests that the large half width is not only due to strong electron-phonon coupling but due to other dominant reasons, such as spacial non-uniformity.

The time decay characteristics of PL also indicate the non-uniform nature of this luminescence. Typically, two kinds of decay characteristics are reported, for which decay times differ by three orders. The very "fast" decay is reported ranging from subnanoseconds to several nanoseconds, depending on temperature $[12,13]$. The most part of CW photoluminescence, however, corresponds to "slow" decay time ranging from several microseconds to some hundred microseconds at room temperature $[14,15]$. This decay further slows down at low temperatures, ranging from several microseconds to several milliseconds. Although the high efficiency $\mathrm{CW}$ luminescence part is represented by slower decay component ( $\mu$ s range), the fast decay component (ns range) corresponds to the quite similar energy dispersion as that of slow component. Both decays show non-exponential behavior which is in apparent coincidence with the non-homogeneous broadening nature of PL. The non-exponential decay is sometimes characterized by the "stretched" exponential, $S(t)=S(0) \exp (-t / \beta)^{\gamma}$, where $\beta$ corresponds to mean lifetime and $\gamma(0<\gamma<1)$ represents the broadening of lifetime. Within "slow" decays, lower energy portion of photoluminescence corresponds to slower decay (small $\beta$ ) and it has narrower distribution of lifetime, i.e. larger $\gamma$ [16].

The temperature dependence of photoluminescence intensity and of peak position shows somewhat strange behavior from a typical band-to-band or impurity-state originated luminescence. The luminescence intensity first increases upon decreasing temperature and further decreases with the decrease in temperature [17]. The temperature at which PL intensity has its maximum depends on sample as well as on excitation intensity. In general case, a typically observable increase in 
intensity towards low temperature in PL is due to decreased thermalization from a trapped carrier or suppressed dissociation of exciton which in turn decreases the probability to non-radiative pathways. In the present case, however, the opposite behavior is observed; the similar temperature dependence to porous Si has been reported in the case of the tail states in $a-\mathrm{Si}$ where excited carriers are localized in space at low temperature [18]. The nature of the luminescence in $a-\mathrm{Si}$ is characterized as spatially separated electron-hole pairs (the time constant $\tau$ of radiative decay is expressed as $\tau^{-1}=\tau_{0}^{-1} \exp \left(-2 r / R_{0}\right)$ ), and the decrease in PL intensity at low temperatures is accounted for by the Auger recombination. The effect becomes dominating when the separation of the recombining electron-hole pair is comparable with the distance to the third carrier. The non-radiative recombination rate determined by this mechanism is dependent on the number of photogenerated carriers and thus the temperature behavior of the PL intensity is dependent on excitation intensity. The observed dependence on excitation power is quite similar to that of $a-\mathrm{Si}$ and this confirms the spatially-separated localized pair nature of porous $\mathrm{Si}$ luminescence [17]. It is interesting that the temperature at which PL intensity begins to decrease $(\approx 100 \mathrm{~K})$ coincides with the observed decrease in conductivity of the film which means that carrier diffusion is of special importance to the luminescence processes. It is naturally speculated from this that at elevated temperature an electron and/or a hole diffuse to final recombination location before recombination and if the diffusion is thermally prohibited then three-particle interaction begins to dominate. The change of peak position upon a change of temperature is also somewhat bizarre as compared with typical photoluminescence [19]; a shift of peak is dependent on sample and energy and no general trend or value can be seen. This is also indicative of non-uniform origin of luminescent substances.

Hydrostatic pressure effects on PL and absorption, which is typically best suited for revealing the nature of bands involved, have been reported but the results come from different institutions [20-22]. Since PL characteristics is strongly dependent on the surface conditions of Si [23], application of pressure medium should induce an important change at the Si surface. In this sense, the pressure coefficient is somewhat meaningless, if the pressure medium induces structural changes of surface. Actually, strong quenching of luminescencies is observed by immersing porous Si into normally used polar pressure transmitting media [24]. In this sense He-gas pressure application is the most mild and reliable way to test the pressure coefficient.

Studies on PL behavior after various treatments have been conducted. A decrease in $\mathrm{PL}$ intensity is typically reported upon annealing up to $200-300^{\circ} \mathrm{C}$ in vacuo, $\mathrm{O}_{2}$ or $\mathrm{N}_{2}$ atmospheres [25, 26]. Such annealing results in a decrease in the surface film coverage by $\mathrm{SiH}_{2}$ [25]. As it is well known, the bulk Si surface after HF dip shows hydrogen-termination, which is known to be very stable. Therefore, similar situation of hydrogen-termination must be a result of anodization in aqueous HF solution and this is indeed verified by the infrared absorption spectroscopy, which reveals the existence of $\mathrm{SiH}_{2}$ bonding. In a simple mind, the dangling bond of $\mathrm{Si}$ acts as a killer center (either through non-radiative or radiative processes) to the visible luminescence by the simple analogy to $a$-Si luminescence, for which 
the dangling bond center cuts down the main luminescence (tail states) intensity even though it produces luminescence in lower energy region.

Changes of PL induced by light irradiation during anodization and by etching/oxidation after anodization have also been reported [27]. In both cases the blue shift of PL together with the observation of smaller size crystallite was obtained. As anodized porous Si typically produces very weak light in the region of $1.4 \mathrm{eV}$ and extended immersion in HF after anodization results in the remarkable PL shift to visible region with an increased intensity. Light irradiation during anodization probably enhances the etching/oxidation. Thus PL shows a blue shift of the peak. The blue shift of luminescence is also observed by electroluminescence in a system which contains aqueous electrode; in other words, during anodic oxidation after anodization luminescence shifts to higher energy with increasing its intensity [28]. In this case (electro)luminescence suddenly ceases because of formation of oxide which prohibits the current injection. A similar blue shift and an increase in intensity is also observable just by dipping in pure water after anodization [29]. Only after $30 \mathrm{~min}$ in water, typical intense, visible photoluminescence is observed whereas as-anodized sample gives very weak luminescence in the infrared region. These experimental results mean that oxidation is quite necessary for obtaining of the visible luminescence. Further oxidation or etching results in further blue shift of the PL spectrum to the green region but together with a decrease in intensity.

\subsection{Anodization and pore structure}

Electrochemistry of pore formation in Si has been studied over 30 years but the basic mechanism of pore formation is not fully understood. It is, however, generally accepted that the holes are required in the initial oxidation steps, in order to release hydrogen to proceed etching and oxidation [5] and this is the reason that light irradiation is necessary for $n$-type substrate.

Although the original proposition was that the structure of porous $\mathrm{Si}$ is a nanometer scale rod or wire system, TEM studies have revealed that the main constituent is microcrystalline $\mathrm{Si}$ embedded in amorphous layer, probably $\mathrm{SiO}_{x}$ [9]. It is interesting that the orientation of these microcrystallites is not lost even in an amorphous layer; it is the same as in a substrate. It is also interesting that no microcrystallites of diameter larger than $1 \mathrm{~nm}$ have been found after oxidation of porous $\mathrm{Si}$, which emits green light [30].

Visible light emitting Si can be made not only from anodization but also from surface damaging and subsequent HF etching/oxidation. The ion beam irradiation damaging produces visible luminescent $\mathrm{Si}$ and these techniques have a strong merit of uniformity and patterning [31]. On the other hand, fabrication of nano-size crystallite by electron beam lithography and related technique has not yet succeeded in producing light-emitting substance. 


\section{Models proposed: theoretical approaches}

Various models have been proposed to explain the mechanism of visible luminescence from porous $\mathrm{Si}$, including (1) size quantization effects due to surface H-terminated Si wire and Si microcluster; (2) existence of specific compounds at the (inner) surface of $\mathrm{Si}$, i.e., siloxene $\left(\mathrm{Si}_{6} \mathrm{O}_{3} \mathrm{H}_{6}\right.$ and its derivatives) or polysilane, $\left(\mathrm{Si}_{2} \mathrm{H}_{2}\right)_{n} ;(3)$ amorphous substances such as $a-\mathrm{Si}: \mathrm{H}, a-\mathrm{SiO}_{x}: \mathrm{H}, a-\mathrm{SiO}_{2}$ and (4) more localized nature of the luminescent center $\mathrm{Si}-\mathrm{HI}$ or Si-O at the (inner) surface. The point is how visible efficient emission could be produced from $\mathrm{Si}$, which is indirect, infrared band-gap material and whose atoms are directly involved in this luminescent entities? It is naturally assumed that this material could involve $\mathrm{Si}$, $\mathrm{H}$ and $\mathrm{O}$, and perhaps $\mathrm{F}$ but the traces of impurity atoms from ambient as well as from Si substrate would not be important.

\subsection{Size quantization}

In a small size semiconductor surrounded by low electron affinity as well as low dielectric constant materials, electron confinement in such a system results in an effective increase in the energy of band gap. The observation of such small wire or rod and dot structures by TEM in high porosity $\mathrm{Si}$ is used to rationalize this model. Furthermore, as described earlier, etching and oxidation of porous $\mathrm{Si}$ lead to the decrease in size of microcrystalline Si together with the blue shift of absorption threshold and PL peak. Independently, synthetic Si or Ge microclusters buried in $\mathrm{SiO}_{2}$ show a size dependent band gap shift and also show broad visible luminescence [32].

The lowest energy level of quantum confined wire structure is represented by $h^{2} / 4 L^{2} m$ ( $L$ - wire width, $m$ - electron mass), assuming parabolic bands semiconductor surrounded by an infinitely high barrier layer. Confinement to small scale crystallite not only increases the effective band gap but also has a large influence on the radiative recombination rate. Bulk Si has an indirect gap but when the wave function is strongly confined, then its Fourier component can be extended and the original indirect nature of $\mathrm{Si}$ could be largely relaxed. Within an effective mass approximation Takagawara calculated the radiative recombination rate and found its large increase when the size of $\mathrm{Si}$ or Ge dots decreases, for which the importance of low dimensional excitonic effect as well as the large dielectric confinement are pointed out [33]. The increase in the radiative recombination rate is strongly dependent on the size, such as $\propto L^{-5}$ [34].

The calculations using the tight-binding approach have also been reported for Si $8 \times 8$ [100] wire and up to 3109 atoms of the Si spherical cluster structure, for which $\mathrm{Si}$ is terminated by hydrogen $[34,35]$. In this case the confinement energy is proportional to $L^{-1.39}$ for $L<4 \mathrm{~nm}$ and these are qualitatively the same results as obtained by the effective mass approach. Proot et al. calculated the radiative recombination rate for Si spherical clusters and compared it with the experimentally observed decay rate [36]. At first sight, the agreement seems to be good in the energy range between $1.6-2.2 \mathrm{eV}$. The calculated decay rate, however, scatters or oscillates on the cluster size because rate is critically dependent on 
the shape of wave function i.e. it depends on size or orientation of clusters. An interesting effect of oxygen termination replaced by hydrogen is reported, for which $\mathrm{Si}_{10} \mathrm{O}_{x} \mathrm{II}_{16-x}$ energy gap decreases upon replacing $\mathrm{H}$ by $\mathrm{O}$ [37].

The first principles calculation has also been reported for wire and spherical structures. For a [100] directed wire structure, anisotropic mass of original $\mathrm{Si}$ is reflected through zone folding and furthermore band mixing results in the direct allowed gap [38]. The existence of the indirect gap with similar energy in conduction band is also confirmed. In this case, $\mathrm{Si}$ is terminated by hydrogen but the $\mathrm{Si}-\mathrm{II}$ state is located far away from the conduction band bottom, indicating that the $\mathrm{Si}-\mathrm{H}$ localized states does not directly participate in the visible luminescence mechanism. The $a b$ initio calculation of $\mathrm{Si}$ cluster up to $\mathrm{Si}_{123}$ has also been reported where the energy gap increases as the size of cluster decreases and correspondingly, the originally forbidden transition becomes weakly allowed $(\tau \approx 1 \mathrm{~ms}$ for $\mathrm{Si}_{123}, \tau \approx 1 \mu \mathrm{s}$ for $\mathrm{Si}_{29}$ ) and finally allowed ( $\tau<10 \mathrm{~ns}$ ) [39].

From these theoretical approaches, fundamental tendency for reducing size of crystal is quite similar; the energy gap increases due to the confinement and can reach the value corresponding to the visible light region when the size becomes less than $3 \mathrm{~nm}$. At the same time, band mixing due to the breakdown of the translational symmetry, zone folding, enhancement of excitonic recombination, and dielectric confinement result in a large optical recombination rate. This could successfully account for the visible emission from porous Si. The size quantization effect is actually observed by the shift of absorption threshold [11]. A large amount of experimental results, however, cannot be explained by the use of only this model. The large PL half width as well as large distribution of luminescent decay time is typically attributed to distribution of different cluster size. Since the radiative decay rate is inversely proportional to fifth power of cluster size, larger size cluster has extremely lower transition probability and to obtain the Gaussian shape $\mathrm{PL}$, one needs a strange profile in size distribution: Also, if a simple quantum confinement is assumed, there should be no large Stokes shift. Furthermore, even though calculated radiative lifetime relatively well accounts for the distribution of luminescent decay time, experimental decay may not necessarily be a radiative lifetime from the luminescent center. It may be modified by non-radiative decay or carrier diffusion effects. The existence of fast decay (subnanoseconds order) requires the presence of extremely small clusters which do not match the observed emission wavelength. The complex mechanism of PL is represented by lower energy absorption threshold than PL energy, however, PL excitation energy is always higher than PL energy. Therefore, the presence of the quantum size effect is probable in porous $\mathrm{Si}$ absorption but luminescence should not be directly connected with this effect.

\subsection{Specific compounds at inner surface; polysilane and siloxene}

Polysilane was proposed as a luminescent substance because inner surface of porous Si was passivated by hydrogen as evidenced by infrared absorption spectroscopy [25]. The fact that with increasing temperature, hydrogen desorption is observed together with decreased PL intensity also supports the model. Polysilane 
is a Si-skeleton material and its one-dimensional and two-dimensional versions were quoted as specific compounds at porous Si surface [40]. The $a b$ initio calculation gives a direct, allowed band gap for 1D polysilane and direct as well as indirect gaps exist for 2D version. Also chemically synthesized polysilane produces visible emission [41]. However, since oxidation of surface seems prerequisite for luminescence, the compounds which contain only $\mathrm{Si}-\mathrm{H}$ bonding are unlikely to be the luminescent substance, although $\mathrm{H}$ plays an important role in porous $\mathrm{Si}$ at least as a terminator of the $\mathrm{Si}$ dangling bond to kill non-radiative pathways.

From the above discussion, $\mathrm{Si}, \mathrm{H}, \mathrm{O}$ must involve in the luminescent substance and then siloxene $\mathrm{Si}_{6} \mathrm{O}_{3} \mathrm{H}_{6}$ and its derivatives are proposed as a candidate. Siloxene has been known to be a quite efficient luminescence molecule even in a solvent as it is typically used as a chemiluminescent indicator for the Ce redox titration. Very strong experimental results have been reported, for which chemically synthesized siloxene shows similar visible luminescence, absorption, and infrared absorption of lattice vibration [42]. Furthermore, porous $\mathrm{Si}$ itself shows chemiluminescence when oxidized with $\mathrm{HNO}_{3}$ [43]. It is quite interesting to note that there are Si magic numbers $(2,6,10, \ldots)$, at which $\mathrm{Si}$ microcluster is stabilized and siloxenc has the same number of Si so that 6 membered Si ring may be preferentially produced during anodization. Theoretical approaches, including semi-empirical as well as ab initio, reveal the band structure of various types of siloxene [44,45]. Siloxene could be thought quite analogous to polysilane and low-dimensional quantum (chemically) confined structure; plane version $\left[\mathrm{Si}_{2} \mathrm{H}(\mathrm{OH})\right]_{3 n}$ is similar to planer polysilane $\left[\mathrm{Si}_{2} \mathrm{H}_{2}\right]_{n}$ and this is $2 \mathrm{D}$, chain version $\left[\mathrm{Si}_{2} \mathrm{H}_{2} \mathrm{O}\right]_{3 n}$ similar to linear polysilane $\left(\mathrm{SiII}_{2}\right)_{n}$ and this is $1 \mathrm{D}$ wire, and six-fold $\left[\mathrm{Si}_{6} \mathrm{H}_{6} \mathrm{O}_{3}\right]_{n}$ is similar to $0 \mathrm{D}$ quantum dot. The detailed band structures are different for different version but the replacement of $\mathrm{H}$ by OH or other monovalent radical can tune the band gap. Also it is pointed out that the role of oxygen lone pair is important to determine the character of the valence band [45].

\subsection{Amorphous structure; a-Si:H, $a-S i O_{x}: H$}

Since amorphous structure is always observed in porous $\mathrm{Si}$, it is natural to consider amorphous layer to be a luminescent substance since $a$-Si and $a-\mathrm{SiO}_{x}$ are known to produce various luminescence in the range from infrared to visible [46]. The luminescence characteristics are quite similar in the sense that it has a broad range of decay times as well as a large half width, for which luminescence is originated from spatially separated electron-hole recombination on the band tail states [18]. $a$-Si:II, which typically produces $\approx 1.4 \mathrm{eV}$ luminescence, could possibly produce visible light if the concentration of $\mathrm{H}$ can be increased. This simultaneously results in inclusion of microcrystalline $\mathrm{Si}$ and this might be closer to the situation with porous $\mathrm{Si}$.

\subsection{Localized center; $\mathrm{Si}-\mathrm{H}, \mathrm{Si}-\mathrm{O}, \mathrm{a}-\mathrm{SiO}_{2}$, with lattice relaxation?}

Since there is an apparent large Stokes shift, large lattice relaxation is proposed to be involved in the luminescent mechanism. The $\mathrm{Si}-\mathrm{H}, \mathrm{Si}-\mathrm{O}$ localized states involve excited electron-hole pairs which are extremely localized at these 
molecules. In amorphous $\mathrm{SiO}_{2}$ typical band tail states are located far above the visible energy region. However, this polar material is known to produce an intrinsic self trapped exciton [47], for which an electron and a hole reside on $\mathrm{Si}$ and $\mathrm{O}$ atom sites and then induce rearrangement of the bond configuration. Therefore, in this sense, the luminescence mechanism in $a-\mathrm{SiO}_{2}$ is a localized one. The necessity of localized pair of porous Si recombination has been pointed out and from this point of view, the large half width is a natural consequence of the large electron-phonon coupling.

\section{Recent experimental results and discussion}

Recently rapid thermal oxidation (RTO) study has been reported, for which, despite the fact that luminescence intensity ceased after annealing up to $400-500^{\circ} \mathrm{C}$, annealing in oxygen up to $700-900^{\circ} \mathrm{C}$ restores the luminescent properties completely or even brighter luminescence is obtained [48]. After complete annealing, no trace of $\mathrm{H}$ in infrared absorption is detected. This fact together with prerequisite of etching/oxidation after as-anodization confirms the primarily importance of oxygen and the necessity of hydrogen involved in the luminescent center. It is interesting to note that after high-temperature annealing a blue shift of PL is observed and this shift appears together with a change in PL intensity; it begins from the red PL and attains maximum intensity at $\approx 1.8 \mathrm{eV}$ and then begins decreasing and high-energy shift stops when the peak reaches the green region $(\approx 2.0 \mathrm{eV})$ with very decreased intensity. This behavior is observed not only during this high-temperature RTO but low-temperature oxidation by etching as well [49]. Further oxidation results in the strong blue PL in the region of $2.7 \mathrm{eV}$ but this does not seem to be the continuous shift from the red region but the blue PL appears suddenly, probably due to rigorous structure or coordination change. It is important to note that, within the red-green shift, TEM observation reveals no trace of crystalline $\mathrm{Si}$ with a size larger than $1 \mathrm{~nm}$ for yellow-green emitting materials [30]. It is also noted that the concentration of Si dangling bond was monitored by ESR during RTO study, for which a steep increase up to $\approx 500^{\circ} \mathrm{C}$ was observed whereas a sudden decrease was observed at higher temperatures when the PL restores its intensity. These facts strongly suggest the importance of oxygen or oxygen-related compound, such as siloxene or $\mathrm{SiO}_{x}$.

Recently infrared luminescence in addition to the visible luminescence was found at low temperatures, which has a quite similar excitation threshold and spectrum to the visible one [17]. This indicates that the IR luminescence is one branch of recombination paths for the excited carriers and the other branch is the visible one. From the optically detected magnetic resonance (ODMR) study of this luminescence, $\mathrm{Si}$-dangling bond, known as $\mathrm{Pb}$ center in $\mathrm{Si} / \mathrm{SiO}_{2}$ interface, was detected from the angular dependence of ODMR splitting. The striking point is that the field dependence shows the dangling bond located in the crystalline environment [50], unlike $a$-Si where the dangling bond is isotropic because of the freedom of $a$-Si network. This is in good agreement with TEM observation. Considering that carriers have to diffuse to find final recombination site (distant pair nature) and IR luminescence occurs during this diffusion, the visible luminescent center is 
located very close to crystalline environment. From these discussions, one possible candidate for the visible luminescence is therefore the surface oxygen containing a compound adjacent to Si microcystallite, such as siloxene or simply $\mathrm{Si}-\mathrm{O}$ bond.

Very recent extended X-ray absorption fine structure (EXAFS) study reports the non-existence of siloxene-like compound in porous $\mathrm{Si}$, for which the nearest neighbor atom from $\mathrm{Si}$ is not $\mathrm{O}$ but $\mathrm{Si}$ [51]. This EXAFS study could deny the abundant coordination of $\mathrm{O}$ next to $\mathrm{Si}$, however, if the amount of oxygen is an order less than the expected concentration of $O$ in siloxene, they cannot be distinguished from the EXAFS. Therefore, oxygen-Si bond is definitely present from IR absorption but its concentration is not enough to form siloxene.

The blue luminescence observed after rigorous oxidation seems very similar to that known in $a-\mathrm{SiO}_{2}$ [46]. The origin of this luminescence is typically attributed to the self-trapped exciton (STE). The PL from STE is also observed in $\mathrm{SiO}_{2}$ crystal where STE mechanism is intrinsic in nature. For this mechanism, the excitation is quite localized since a hole resides on $\mathrm{O}$ and an electron on $\mathrm{Si}$ which then induces lattice rearrangement [47]. There is no report for visible luminescent STE in $\mathrm{SiO}_{2}$ but $\mathrm{Si}-\mathrm{O}$ defect state can be much different if it is located at the surface rather than in the crystal or amorphous network, since the oxygen coordination number as well as freedom of structure change are much different. As already mentioned before, siloxene as well as porous Si chemiluminesce are present in a solvent. Typically solvation leads to dispersion of the excitation energy to adjacent molecule or to lattice. But the nature of chemiluminescence suggests that the excitation can be very localized, probably owing to the existence of some thermal barriers to overcome for electron or hole.

From these considerations it is proposed that the visible luminescence is originated from the electron-hole recombination at localized surface $\mathrm{Si}-\mathrm{O}$ bond adjacent to $\mathrm{Si}$ microcrystallite. However, as in $\mathrm{SiO}_{2}$, efficient excitation must come from much higher energy. The excitation in porous $\mathrm{Si}$, therefore, comes from adjacent microcrystalline Si which absorbs light first. This microcrystalline Si has a large enough band gap due to the size quantization effect and once excited, an electron and/or a hole diffuse to be trapped on the Si-O bond. Upon excitation, this bond undergoes large lattice relaxation which easily accounts for the large half width of PL. This mechanism explains most of experimental results reported but still experimental substantiation should be made, possibly by ODMR or other structure sensitive characterization of electronic states. The possible structure and mechanism of self trapping to produce the visible red luminescence is unknown at present. However, the lattice rearrangement should be much easier in these small substances which emphasizes the fundamental importance of lattice relaxation in a low dimensional system.

\section{Acknowledgments}

The author would like to thank Y. Mochizuki, N. Hamada, A. Oshiyama, Y. Ochiai, N. Ookubo for discussion and R. Lang for encouragement. 


\section{References}

[1] L.T. Canham, Appl. Phys. Lett. 57, 1046 (1990).

[2] V. Lehmann, U. Gosele, Appl. Phys. Lett. 58, 856 (1991).

[3] J.C. Vial, A. Bsiesy, F. Gaspard, R. Herino, M. Ligeon, M. Fuller, R. Romestain, R.M. MacFarlane, Phys. Rev. B 45, 14171 (1992).

[4] P.J. Dean, J. Lumin. 1/2, 398 (1970).

[5] R.L. Smith, S.D. Collins, J. Appl. Phys. 71, R1 (1992).

[6] H. Koyama, M. Araki, Y. Yamamoto, N. Koshida, Jpn. J. Appl. Phys. 30, 3606 (1991).

[7] N. Koshida, H. Koyama, Mater. Res. Symp. Proc. 256, 219 (1992).

[8] L. Wang, M.T. Wilson, M.S. Goorsky, N.M. Haegel, Mater. Res. Symp. Proc. 256, 73 (1992).

[9] A. Nishida, K. Nakagawa, H. Kakibayashi, T. Shimada, Jpn. J. Appl. Phys. 31, L1219 (1992).

[10] J.M. Perez, J. Villalobos, P. McNeill, J. Prasad, R. Cheek, J. Kelber, J.P. Estrera, P.D. Stevens, R. Glosser, Appl. Phys. Lett. 61, 563 (1992).

[11] Y. Udo, Y. Kanemitsu, Y. Masumoto, unpublished.

[12] S. Gardelis, J.S. Rimmer, P. Dawson, B. Hamilton, R.A. Kubiak, T.E. Whall, E.H.C. Parker, Appl. Phys. Lett. 59, 2118 (1991).

[13] T. Matsumoto, M. Daimon, T. Futagi, H. Mimura, Jpn. J. Appl. Phys. 31, L619 (1992).

[14] M. Kondo, unpublished.

[15] G.W. 't Hooft, Y.A.R.R. Kessener, G.L.A. Rikken, A.H.J. Venhuizen, Appl. Phys. Lett. 61, 2344 (1992).

[16] N. Ookubo, H. Ono, Y. Ochiai, Y. Mochizuki, S. Matsui, Appl. Phys. Lett. 61, 940 (1992).

[17] Y. Mochizuki, M. Mizuta, Y. Ochiai, S. Matsui, N. Ookubo, Phys. Rev. B 46, 12353 (1992).

[18] R.A. Street, in: Semiconductors and Semimetals, Vol. 21, Academic Press, New York, part B, p. 197.

[19] Z.Y. Xu, M. Gal, M. Gross, Appl. Phys. Lett. 60, 1375 (1992).

[20] W. Zhou, M. Dutta, H. Shen, J.F. Harvey, R.A. Lux, C.H. Perry, R. Tsu, N.M. Kalkhoran, F. Mamovar, in: Proc. Int. Conf. Phys. Semicond., Beijing, 1992, World Scientific, Singapore, p. 1479.

[21] J. Camassel, E. Massone, S. Lyapin, J. Allegre, P. Vincente, A. Foucaran, A. Raymond, J.L. Robert, in: Proc. Int. Conf. Phys. Semicond., Beijing, 1992, World Scientific, Singapore, p. 1463.

[22] N. Ookubo, Y. Matsuda, N. Kuroda, unpublished.

[23] A. Nakajima, T. Itakura, S. Watanabe, N. Nakayama, Appl. Phys. Lett. 61, 46 (1992).

[24] J.M. Lauerhaas, G.M. Credo, J.L. Heinrich, M.J. Sailor, Mater. Res. Symp. Proc. 256, 137 (1992).

[25] M.B. Robinson, A.C. Dillon, D.R. Haynes, S.M. George, Mater, Res. Symp. Proc. 256, 17 (1992). 
[26] M.A; Tischler, R.T. Collins, J.H. Stathis, J.C. Tsang, Appl. Phys. Lett. 60, 639 (1992).

[27] S. Shih, K.H. Jung, D.L. Kwong, M. Kovar, J.M. White, Appl. Phys. Lett. 62, 1904 (1993).

[28] A. Halimaoni, C. Oules, G. Bomchil, A. Bsiesy, F. Gaspard, R. Herino, M. Ligeon, F. Muller, Appl. Phys. Lett. 59, 304 (1991).

[29] T. Tamura, A. Takazawa, M. Yamada, unpublished.

[30] Y. Ochiai, N. Ookubo, H. Watanabe, S. Matsui, Y. Mochizuki, H. Ono, S. Kimura, T. Ichihashi, Jpn. J. Appl. Phys. 31, L560 (1992).

[31] Y. Ochiai, T. Ichihashi, H. Ono, Y. Mochizuki, unpublished.

[32] H. Takagi, H. Ogawa, Y. Yamazaki, A. Ishizaki, T. Nakagiri, Appl. Phys. Lett. 56, 2379 (1990).

[33] T. Takagawara, K. Takeda, Phys, Rev. B 46, 15578 (1992).

[34] G.D. Sanders, Y.-C. Chang, Phys. Rev. B 45, 9202 (1992).

[35] S.Y. Ren, J.D. Dow, Phys. Rev. B 45, 6492 (1992).

[36] J.P. Proot, C. Delerue, G. Allan, Appl. Phys. Lett. 61, 1948 (1992).

[37] Y. Kito, R. Kumar, H. Inuzuka, K. Shigematsu, K. Hara, unpublished.

[38] T. Ohno, K. Shiraishi, T. Ogawa, Phys. Rev. Lett. 69, 2400 (1992).

[39] T. Uda, M. Hirao, J. Shimada, unpublished.

[40] K. Takeda, K. Shiraishi, Phys. Rev. B 39, 11028 (1989).

[41] D.J. Wolford, B.A. Scott, J.A. Reiner, J.A. Bradley, Physica B 117/118, 920 (1983).

[42] M.S. Brandt, H.D. Fuchs, M. Stutzmann, J. Weber, M. Cardona, Solid State Commun. 81, 307 (1992).

[43] P. McCord, S.-L. Yau, A.J. Bard, Science 257, 68 (1992).

[44] P. Deak, M. Rosenbauer, M. Stutzmann, J. Weber, M.S. Brandt, Phys. Rev. Lett. 69, 2531 (1992).

[45] K. Takeda, K. Shiraishi, Solid State Commun. 85, 301 (1993).

[46] K. Tanimura, C. Itoh, N. Itoh, J. Phys. C 21, 1869 (1988).

[47] W. Hayes, M.J. Kane, O. Salminen, R.L. Wood, S.P. Doherty, J. Phys. C 17, 2943 (1984).

[48] V. Petrave-Koch, T. Muschik, A. Kux, B.K. Meyer, F. Koch, Appl. Phys. Lett. 61, 943 (1992).

[49] K. Motoi, T. Ohta, O. Arakaki, T. Ito, A. Hiraki, unpublished.

[50] Y. Mochizuki, M. Mizuta, unpublished.

[51] S.L. Friedman, M.A. Marcus, D.L. Adler, Y.-H. Xie, T.D. Hrris, P.H. Citrin, Appl. Phys. Lett. 62, 1934 (1993). 